Classification

Physics Abstracts

$61.16 \mathrm{ch}-68.35 \mathrm{G}$

\title{
Contact AFM on soft surfaces: Elasticity and friction effects
}

\author{
Christian Frétigny and Marie-Claire Boisset
}

CNRS URA 1428, ESPCI/LPQ, 10 rue Vauquelin, F-75231 Paris Cedex 05, France

(Received July 4; accepted October 26, 1994)

\begin{abstract}
Résumé. - Nous montrons des images obtenues en AFM de contact d'un film recuit de billes de latex de $5^{\circ}$ de température de transition vitreuse. La surface présente un arrangement hexagonal de sphères aplaties. Le contraste dépend fortement de la force appliquée et de la direction de balayage : les sphères apparaissent soit concaves soit convexes. Une analyse des frottements montre distinctement deux régimes de fonctionnement du microscope. Pour de forts grossissements, la pointe est profondément enfoncée dans le matériau et les images ne reflètent pas la topographie de la surface, mais semblent caractéristiques de ses propriétés mécaniques. Pour de plus grands domaines de balayage, le frottement est de type classique et la résolution est bonne. Ces résultats permettent de préciser les limitations de la technique pour des matériaux mous.
\end{abstract}

\begin{abstract}
We present contact AFM images of an annealed film of latex balls of $5^{\circ}$ glass transition temperature. Surface appears to be constituted of an hexagonal arrangement of flatten spheres. Contrast is seen to depend very much on the applied load and on the scan direction: spheres appear concave or convex according to these parameters. An analysis of the friction clearly shows that there are two regimes for imaging. For high magnifications, the tip is deeply intruded in the material and images do not reflect the topography of the surface but seem to be characteristic of its mechanical properties. For higher scan domains, friction is of classical type and resolution is good. These results permit to clarify the limitations of the technique applied on soft materials.
\end{abstract}

\section{Introduction.}

In imaging soft materials using contact AFM, it has been noted that sometimes non-topographic results or low resolution can be obtained [1,2]. It is of particular importance to analyse the limitations of the technique for these materials, specially for biology and polymer science studies.

One of the causes which can affect the results obtained using this microscopy is adhesion and frictional forces which makes the deflection of the cantilever not only due to topographic details. It has been shown that one can take advantage of friction [3,4] and adhesion [5] to obtain images by AFM. We show here that adhesion and friction are the main reasons of the poor quality of contact AFM on soft materials at high magnifications. Conversely, AFM offers a unique opportunity to analyse these mechanical properties for very small contact area. 
As a model surface, we used latex films, with well characterised details on the surface. Dependence of the images on applied load and direction of scan is presented. An analysis of friction for different scan frequencies and loads evidences that a threshold in scan size exists below which no reliable image of the sample can be obtained.

\section{Experimental.}

The film is made from an aqueous dispersion of polymer latex particles. The particles are spherical droplets made of a hydrophobic polymer core surrounded by a hydrophilic layer. The average particle diameter, as measured by electron microscopy, was found to be about $120 \mathrm{~nm}$, and the corresponding size distribution is very narrow.

The particles are made using a semicontinuous procedure where monomers are continuously added to an initial start-up. The composition of the reaction mixture was as follows: styrene, $36.7 \%$, butyl acrylate, $60 \%$, acrylic acid, $3.3 \%$, initiator, ammonium persulfate. A small amount of anionic surfactant was added to control the number and size of the growing particles.

The particle core is made of a statistical copolymer of styrene and butyl acrylate. The glass transition temperature of this copolymer is around $5^{\circ} \mathrm{C}$. Hence at room temperature, this copolymer is a viscoelastic amorphous material The core of each latex particle is surrounded by a thin hydrophilic layer made of acrylic acid sequences which are copolymerized with the core polymer. These acid groups have been neutralised at $\mathrm{pH}=8.0$, hence they are charged and generate long range repulsion between particles, thereby stabilising the dispersion.

Films of about $2 \mathrm{~mm}$ thickness are obtained by solvent evaporation. Small angle neutron scattering and electron microscopy on similar films have shown that a cellular structure remains in the bulk material. Rhombic dodecahedrons, corresponding to fcc packed soft spheres were observed [6].

Images were obtained using commercial AFM instruments [7]. We used commercial silicon nitride cantilever with pyramidal tip and quoted spring constant of $0.58 \mathrm{~N} \cdot \mathrm{m}^{-1}$. All images were obtained in constant force mode. Plane subtraction are the only processing used for the images presented here.

\section{Results and discussion.}

Figure 1 presents an analysis of the normal load effects on the film. An hexagonal arrangement is clearly seen on all the pictures. This cellular structure corresponds to the (111) plane of the reminiscent fcc arrangement observed by neutron diffraction [6]. However, in figure $1 \mathrm{~b}$ recorded at minimal load, the structur 2 appears to be composed of concave flatten spheres, contrarily to other pictures. This image was rather difficult to acquire since, for this low load, dynamic adhesion force was almost cancelled by the force exerted by the cantilever making the system quite unstable. The inverted contrast is seen to increase as the applied load increases. At high loads, figure 1d, surface appears as being damaged by scanning, but subsequent analysis at lower loads, restores the quality. Non-contact and tapping mode experiments showed that the surface is actually made of concave flatten spheres. Contact AFM of this soft sample is thus very sensitive to the applied normal load. It is possible to understand this effect if the equivalent spring constant of the material is comparable to that of the cantilever for low frequencies. On the images, we can appreciate the resolution to be about several tens of nanometers. This implies that the contact radius, a, has the same order of magnitude. Assuming that the Johnson-Kendall-Roberts [8] model (JKR) remains valid during sliding friction, the effective spring constant of the material is given by the 


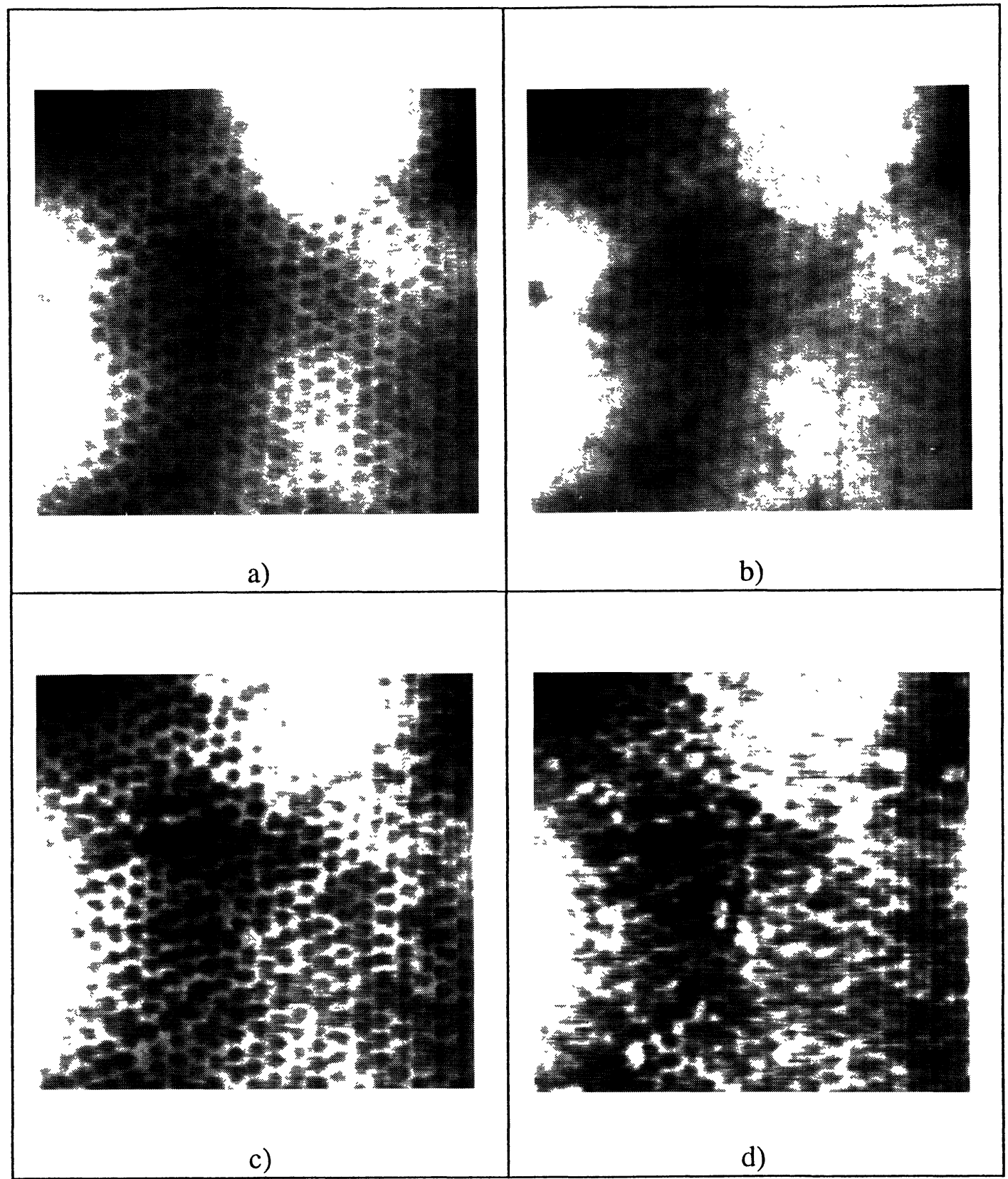

Fig. 1. - AFM of the same area of a latex film in contact mode for different applied loads. a) is a typical image. The surface appears to be composed of a compact arrangement of convex particles. b) is recorded at minimal load. Notice that the contrast is inverted compared to other images. c) is obtained for higher loads. The contrast is amplified compared to a). In d), for even higher load, surface appears as damaged but subsequent analysis at lower load restores images as in a) or b). (Scan size: $2.5 \times 2.5 \mu \mathrm{m}^{2}$ ).

relation $k^{*}=3 / 2 K . a$ [9] for a spherical tip end. With an elastic parameter $K$ of $1 \mathrm{Mpa}$ and neglecting interfacial forces, $k^{*}$ is less than or about $0.1 \mathrm{~N} \cdot \mathrm{m}^{-1}$. This value is equivalent to that 
of the cantilever and the material can be continuously deformed by increasing normal load. For higher frequencies, as in tapping mode, due to its viscoelastic behaviour this material appears to be hard and its surface is not affected by the constraints exerted by the tip. It should be noted that a similar contrast inversion has also been observed on DNA strands adsorbed on mica when humidity over the sample was modified [2].

We also could obtain contrast inversion by simultaneously recording images for scanning directions back and forth along the cantilever for low applied loads. Moreover, contrast is seen to be continuously inverted by rotating the scanning angle. Since the frictional forces are the only differences between both directions, AFM images of this material are seen to be very sensitive to friction. We also observed that load sensitivity was dependent on the position of laser spot on the cantilever. This last point confirms the role of friction forces in the imaging process since the relative weight of normal and lateral forces depends in deflexion mechanism depends on the position of analysis.

An explanation for this phenomenon could be that frictional forces make the cantilever buckling, and corresponding deflexion is interpreted by the feed-back loop as if it were due to topography. It changes the height of the sample which modifies in turn the normal load. Due to sensitivity of the sample to the normal load, this can cause contrast inversion. This explanation holds also in "deflexion mode", as we observed it, where there is almost no gain for the feed-back loop, since it remains a slow feed-back for the height. Other explanations can be found in the rather complicates behaviour of the cantilever under normal and friction forces $[10,11]$.

To better understand friction at this scale, we analyse the variations of $z$ hysteresis between back and forth scanning directions. The difference in piezo height between the two scanning directions along the cantilever direction has been shown to be representative of the longitudinal force exerted on the tip [1, 2]. Quantitative analysis of friction depends on the position of the laser beam on the cantilever and there is no perfect linearity of the signal on the tangential forces [12]. However, for the purpose of the present analysis and on a qualitative basis, it is sufficient to understand the difference between the heights in both directions as being primarily due to friction. In figure 2 are presented the variations of the hysteresis value with the scan size with a frequency $\nu=4.34 \mathrm{~Hz}$. It is clear from this figure that there exist two regions separated by a abrupt transition at about $700 \mathrm{~nm}$. This behaviour is rather similar to the stick-slip motion , "stiction effect", observed in reference [12] when the $x$ position of the sample is modulated under the tip when scanning. We observed that the transition presents an hysteretic behaviour. Figure 2 corresponds to data obtained in increasing scan sizes.

Before the threshold, no topographic information could be recorded: The scans are featureless. The tip is deeply intruded in the sample, the contact area is large and there is no resolution. Adhesion is the main applied force: there are almost no modifications of the $z$ hysteresis when applied normal load is changed. To better understand the behaviour at low scan size, we can evaluate the penetration of the tip in the material at rest and with zero applied force in the JKR model [8]. Approximating the tip with a cone with $45^{\circ}$ half-angle , using the formulae for this geometry [14], with the elastic constant already given above, and a Dupré's energy of $50 \mathrm{~mJ} \cdot \mathrm{m}^{-2}$, we obtain a penetration depth of about $400 \mathrm{~nm}$ due to interfacial energy. A certain size is thus necessary to make the tip go out its hole. Direct experimental confirmation of this penetration of the tip was recently obtained [10]. Figure 3 shows the ratio of the difference in heights to the scan size versus the scan frequency. A power law, with an exponent of about 0.5 is characteristic of the dissipation losses of polymers near their glass temperature transition as well as of the friction effects [14]. No clear model for this characteristic behaviour is available until now, but we have observed it on other rubber-like materials [10].

Above the threshold, resolution is suddenly better, about few tens of nanometers, which implies a very much reduced contact area. Frictional behaviour is now clear since, as presented in figure 4, 


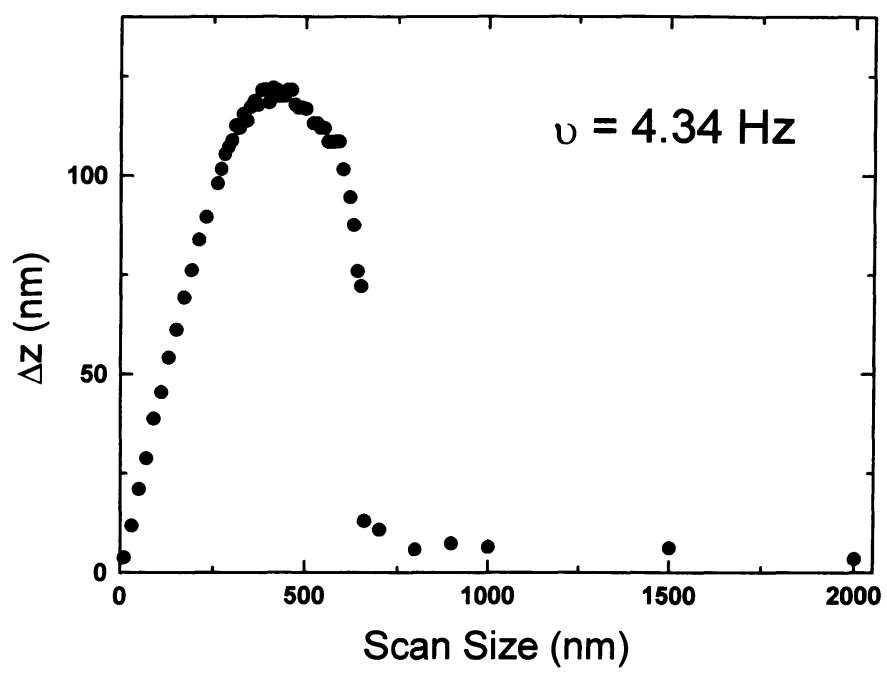

Fig. 2. - Difference in heights for both scan directions (back and forth along the cantilever direction) for different scan sizes. An abrupt transition separates two distinct regions. Data were measured in increasing scan sizes with a frequency $\nu=4.34 \mathrm{~Hz}$. An hysteresis exists for the transition (not shown).

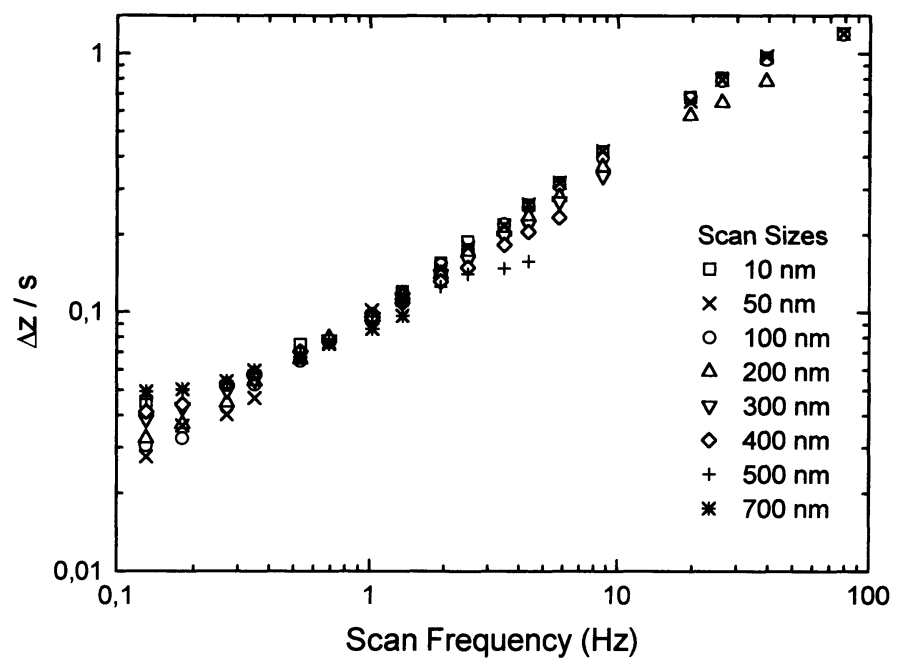

Fig. 3. - Ratio of the hysteresis value of the sample height $(\Delta z)$ to the scan size $s$, plotted against the scan frequency $\nu$. This ratio is seen to be almost independent on the scan size and follows approximately a $\nu^{0.5}$ power law for almost three decades in frequency and over all the range of scan sizes in the first region.

hysteresis is shown to be proportional to the applied load. This is typical of the simple friction law, with a friction coefficient estimated at about 0.2 . This value is not very precise since it depends crucially on the geometrical characteristics of the tip which are not precisely known. Moreover, we observed that friction is almost independent on the scan frequency as expected for ordinary friction. 


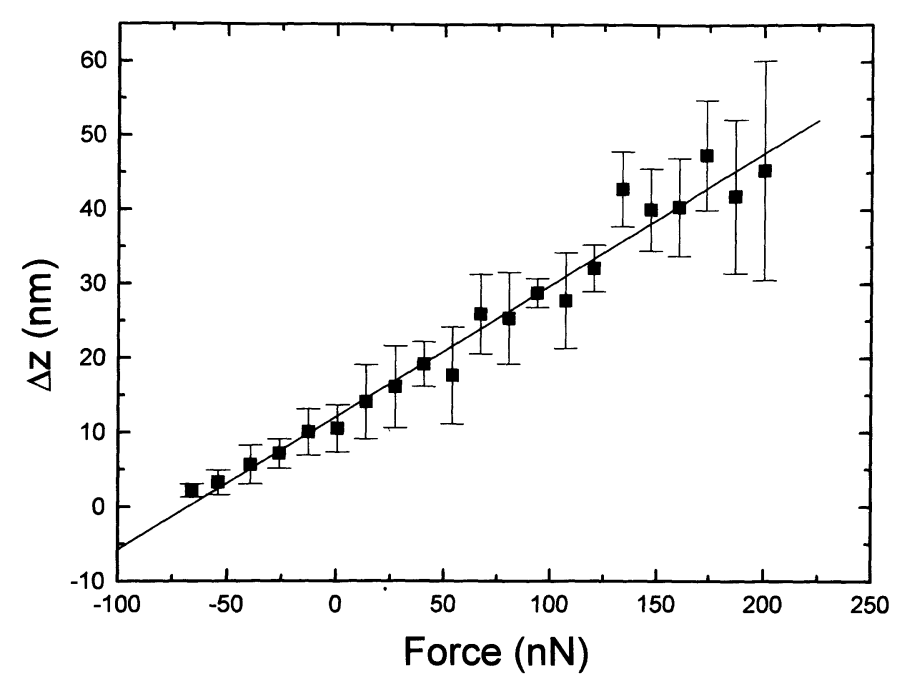

Fig. 4. - Variation of the hysteresis value of the sample height $(\Delta z)$ versus the applied normal load. Linear dependence is characteristic of a normal friction behaviour. Notice that the origin of the abscissa is arbitrary and the value of the force is given using the commercially quoted value for the cantilever $\left(0.58 \mathrm{~N} \cdot \mathrm{m}^{-1}\right)$. Deduced friction coefficient is about 0.2 .

\section{Conclusion.}

We presented here an AFM study of latex film of low $T_{\mathrm{G}}$. The surface reveals hexagonal arrangements of flatten spheres. However contrast is seen to depend very much on experimental conditions: scan direction and applied load. Contrast can be continuously changed and can even be inverted. We observed that no topographical image could be obtained below an abrupt magnification threshold. Below the transition, the tip deeply penetrates into the sample, leading to high contact area, lateral forces exerted by the sample to the tip seems to be characteristic of the mechanical properties of the material. After the transition the contact area is very much reduced and the tip "surfs" on the surface: it is possible to obtain high resolution images. This behaviour has been reproduced on other soft materials and analysis of the parameters like temperature, cross- linking, surface energy is on progress to better understand the phenomenon. Adhesion and friction effects seems to be one of the limitations for the application of contact AFM on soft materials: Reliable height measurements, relative contrasts and high magnifications are not always possible on soft samples. Conversely, because these effects seem to be linked to adhesion and friction AFM offers a unique opportunity to study these properties at nanometer scale. Due to their simple, adaptable and well characterised structure, latex films can be used as a model for these studies.

\section{Acknowledgements.}

We acknowledge M. Barquins and J.P. Aimé for many valuable discussions. 


\section{References}

[1] see for example Radmacher M., Tillmann R.W., Fritz M., Gaub H.E., Science 257 (1994) 1900; Burnham N.A., Colton R., Pollock H.M., J. Vac. Sci. Technol. A9 (1991) 2548.

[2] Thundat T., Warmack R.J., Alison D.P., Lourenco A.J., Ferrel T.I., Bottomley L.A., J. Vac. Sci. Technol. A10 (1992) 632.

[3] Overney R.M., Meyer E., Frommer J., Brodbeck D., Luthi R., Howald L., Gundtherödt H.-J., Fujihara M., Takano H., Gotoh Y., Nature 359 (1992) 133.

[4] Overney R.M., Meyer E., Frommer J., Brodbeck, D., Luthi R., Howald L., Gundtherödt H.-J., Langmuir 10 (1994) 1281.

[5] Van der Werf K.O., Putman C.A., de Grooth B.G., Greve J., Appl. Phys. Lett. 65 (1994) 1195.

[6] Chevalier Y., Pichot C., Graillat C., Joanicot M., Wong K., Maquet J., Lindner P., Cabane B., Colloid Polym. Sci. 270 (1992) 270;

Joanicot M., Wong K., Richard J., Maquet J., Cabane B., Macromol. 26 (1993) 3168.

[7] Nanoscope II and Nanoscope III, Digital Instruments, Santa Barbara, California U.S.A.

[8] Johnson K.L., Kendall K., Roberts A.D., Proc. R. Soc. London 324 (1971) 301.

[9] Barquins M., Maugis D., J. Adhes. 14 (1981) 63.

[10] Fretigny C., to be published.

[11] Aime J.P., Elkaakour Z., Kurely J., Bouhacina T., Odin C., to be published.

[12] Warmack R.J., Zheng X.Y., Thundat T., Allison D.P., Rev. Sci. Instrum. 65 (1994) 394;

Neumeister J.M., Ducker W.A., Rev. Sci. Instrum. 65 (1994) 2527.

[13] Göddenhenrich T., Müller S., Heiden C., Rev. Sci. Instr. 65 (1994) 2870.

[15] Barquins M., In Wear and Friction of elastomers; R. Denton and M.K. Keshavan Eds. (ASTM: Philadelphia, 1992) 82. 